\title{
Microstructure investigations of the phase boundaries in the Bridgman TRIP steel crystal
}

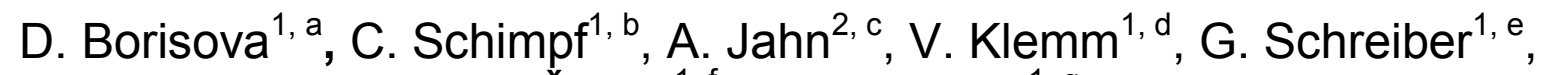 \\ D. Šmek ${ }^{1, f}$ and D. Rafaja ${ }^{1, g}$ \\ ${ }^{1}$ Institute of Materials Science, TU Bergakademie Freiberg, Gustav-Zeuner-Str. 5, \\ D-09599 Freiberg, Germany \\ ${ }^{2}$ Institute for Iron and Steel Technology, TU Bergakademie Freiberg, Leipziger Str. 34, \\ D-09599 Freiberg, Germany \\ aborissov@ww.tu-freiberg.de, ${ }^{b}$ schimpf@ww.tu-freiberg.de, ${ }^{c}$ andreas.jahn@iest.tu-freiberg.de, \\ dklemm@ww.tu-freiberg.de, e schreiber@ww.tu-freiberg.de, 'simek@fzu.cz, ${ }^{\mathrm{g}}$ rafaja@ww.tu- \\ freiberg.de
}

Keywords: Bridgman growth, TRIP steel, phase distribution, microstructure defects, micro-twins, austenite, ferrite, EBSD, XRD.

\begin{abstract}
Formation of microstructure defects at the phase boundaries in TRIP steels was investigated with the aid of microstructure analysis on a TRIP steel crystal, which was grown by the Bridgman technique. The microstructure studies comprised scanning electron microscopy (SEM), X-ray diffraction (XRD), electron backscatter diffraction (EBSD), transmission electron microscopy (TEM) and transmission electron microscopy with high resolution (HRTEM). Initial XRD measurements revealed that the crystals under study consist of austenite and ferrite with extremely strong preferred orientations. Subsequent XRD pole figure measurements and EBSD scans have shown that the orientation relationship between austenite and ferrite can be described by the Nishiyama-Wassermann model. For a detailed description of the microstructure of the Bridgman crystal, the orientation distribution of crystallites within the individual phases was investigated using the XRD reciprocal space mapping and the rocking curve measurements. These experiments have shown that the density of microstructure defects is much lower in ferrite than in austenite. The direct information about the defect structures at the phase boundaries between austenite and ferrite was obtained from the TEM micrographs, which revealed complicated micro-twin structures at the boundaries between the neighbouring phases. HRTEM discovered very narrow stripes of ferrite embedded in austenite that were regarded as a source of the microstructure defects in austenite.
\end{abstract}

\section{Introduction}

The transformation induced plasticity (TRIP) plays an important role in the microstructure based design of modern steels with high yield strength, see, e.g., [1-3]. However, the microstructure based design of the TRIP steels is impossible without understanding the consequences of the deformation processes, which take place within individual phases and at the phase boundaries. The first step in understanding the processes is a detailed microstructure analysis of the TRIP steels, see, e.g., [4, 5].

Different crystal structures of individual phases being in contact at the phase boundaries in a TRIP steel crystal are a source of the lattice misfit, which is responsible for formation of local strain fields. The orientation relationship between the face-centred cubic (fcc) austenite and the bodycentred cubic (bcc) ferrite in iron and its alloys was described in several models [6 - 9], one of them being the Nishiyama-Wassermann model, which was successfully applied to describe the orientation relationship in our samples. Within the Nishiyama-Wassermann model, the parallel lattice planes are $(111)_{\mathrm{fcc}} \|(011)_{\mathrm{bcc}}$, the parallel directions $[-211]_{\mathrm{fcc}} \|[01-1]_{\mathrm{bcc}}[8,9]$. If the local lattice strain (at the phase boundaries) exceeds a threshold limit value, the plastic deformation starts. Its typical consequences are the formation and movement of microstructure defects as well as the local lattice rotations. The microstructure defects, such as dislocations, stacking faults and twins, 
will develop preferably in the austenitic phase because the austenite with the fcc structure has a lower stacking fault energy than the ferrite with the bcc structure [10].

In this work, we describe the formation of microstructure defects at the phase boundaries in a TRIP steel crystal which was grown by a Bridgman technique. The investigation of the microstructure defects comprised scanning electron microscopy (SEM), X-ray diffraction (XRD), electron backscatter diffraction (EBSD), transmission electron microscopy (TEM) and transmission electron microscopy with high resolution (HRTEM). The SEM analysis yielded an overview about the phase distribution within the Bridgman steel crystal. The XRD pole figure measurements and the EBSD measurements were used to clarify the orientation distributions in the crystal. Detailed microstructure analysis that was focussed on the description and visualisation of the microstructure defects was performed using the reciprocal space mapping (RSM) in XRD, TEM and HRTEM.

\section{Experimental details}

The TRIP steel crystal containing $17.2 \mathrm{wt} \% \mathrm{Cr}, 5.5 \mathrm{wt} \% \mathrm{Ni}, 5.8 \mathrm{wt} \% \mathrm{Mn}, 1-2 \mathrm{wt} \% \mathrm{Si}$ and $0.04 \mathrm{wt} \%$ $\mathrm{C}$ was grown using the Bridgman technique [11]. For the microstructure analysis, a part of the Bridgman crystal was cut, ground and mechanically polished. In order to minimize the straininduced surface deformation, which arose during the mechanical polishing, a subsequent electrochemical polishing was applied, particularly for samples intended for XRD and EBSD experiments. For the SEM analysis, the surface of the samples was etched selectively using a Beraha-II solution. TEM sample preparation has been carried out using ion thinning of thin slices.

The XRD pole figures were measured on a Seifert XRD 3000 PTS diffractometer $(\mathrm{Cu}$ radiation, sealed X-ray tube operated at $40 \mathrm{kV}$ and $30 \mathrm{~mA}$ ). The symmetrical Bragg-Brentano XRD measurements were carried out using a URD-6.5 diffractometer from the Freiberger Präzisionsmechanik (FPM). The reciprocal space maps were measured selected reciprocal space points with a high resolution triple-axis X-ray diffractometer, which was based on a URD-6 goniometer from FPM that was equipped with a sealed X-ray tube with $\mathrm{Cu}$ anode, with a perfect (111)-oriented $\mathrm{Si}$ crystal monochromator in the primary beam and with a perfect (111)-oriented Si analyser. The EBSD data and SEM images were taken on the ZEISS LEO 1530 FESEM high resolution scanning electron microscope at the acceleration voltage of $20 \mathrm{kV}$. Transmission electron microscopy (TEM) and transmission electron microscopy with high-resolution (HRTEM) were carried out on an analytical JEOL HRTEM JEM $2010 \mathrm{FEF}$ at an acceleration voltage of $200 \mathrm{kV}$. The analytical HRTEM was working with an ultra-high resolution objective lens $\left(\mathrm{C}_{\mathrm{s}}=0.5 \mathrm{~mm}\right)$ and an in-column energy filter.

\section{Results and discussion}

During the solidification of the Cr-Mn-Ni TRIP steel in the Bridgman process, bcc ferrite and fcc austenite developed. The distribution of the phases is illustrated by the SEM micrograph shown in Fig. 1. The larger fraction of the crystal consists of austenite, which is interspaced by a network of residual ferrite. The amount of ferrite in the Bridgman crystal was approximately $10-15 \%$.

The XRD pole figures measured on the diffraction lines 111, 200 and 220 of austenite and on the diffraction lines 110, 200 and 222 of ferrite (Figs. 2 and 3, respectively) confirmed that the individual phases have almost single-crystalline character. The principal crystallographic orientations of austenite and ferrite with respect to the sample surface were $\langle 33-1\rangle$ and approximately $\langle 001\rangle$, respectively, as obtained from the simulation of the pole figures for various crystal orientations. Furthermore, it follows from the XRD pole figures (Figs. 2 and 3) that the lattice planes $(111)_{\text {fcc }}$ and $(011)_{\text {bcc }}$ lay parallel to each other. With the aid of the complete pole figures calculated from the EBSD measurements (Figs. 2 and 3), the mutual orientation of ferrite and austenite was assessed to correspond to the Nishiyama-Wassermann model $[8,9]$. This orientation relationship is better visible in the pole figures that were calculated from the EBSD measurements than in the pole figures that were directly measured using XRD. A reason for the "incomplete" $\mathrm{XRD}$ pole figures is a limited sample inclination during the XRD pole figure measurement. The 
maximum inclination of the sample lays at $75^{\circ}$, thus the poles located at the border of the pole figures cannot be measured directly.

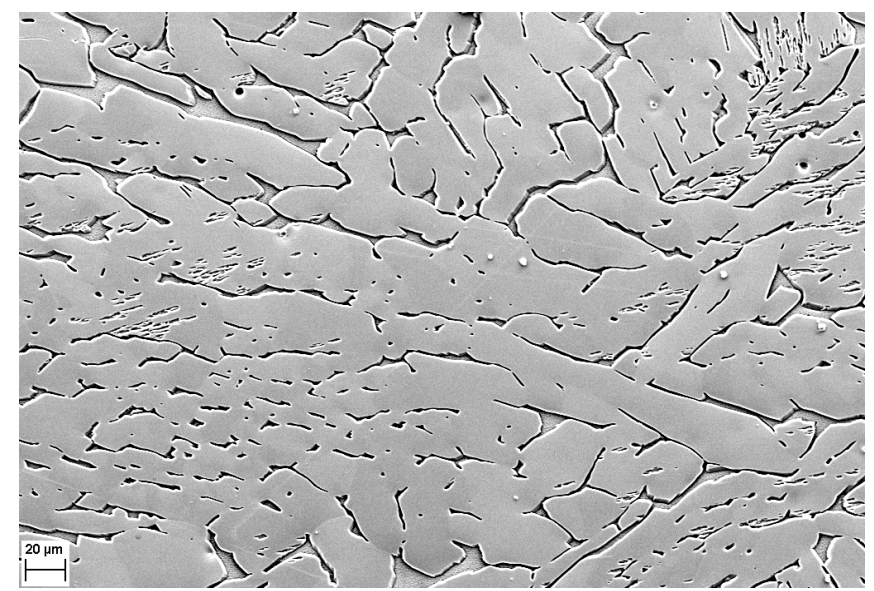

Fig.1. Microstructure overview of the phase distribution in the Bridgman crystal as seen by SEM. The austenite areas are light gray, the network of the residual ferrite is dark gray.

(001)fcc

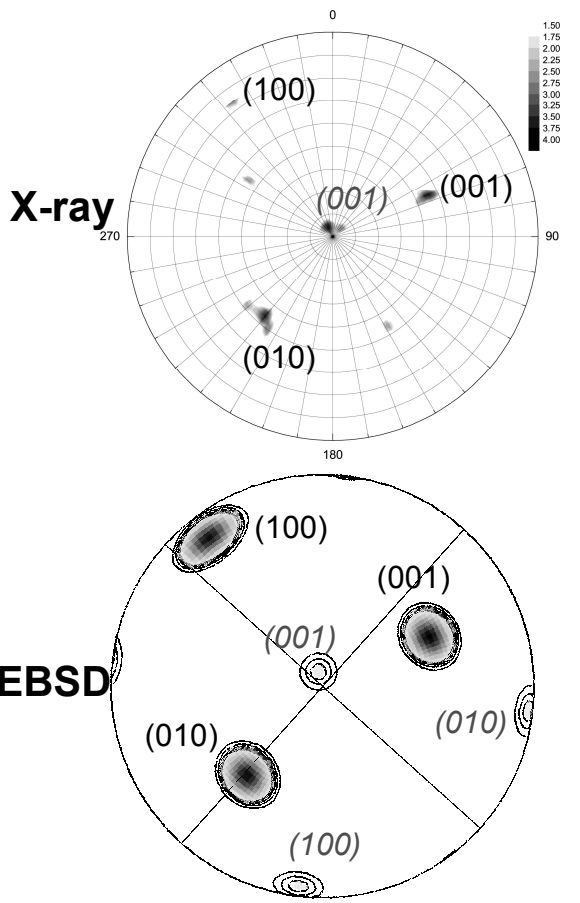

(101)fcc

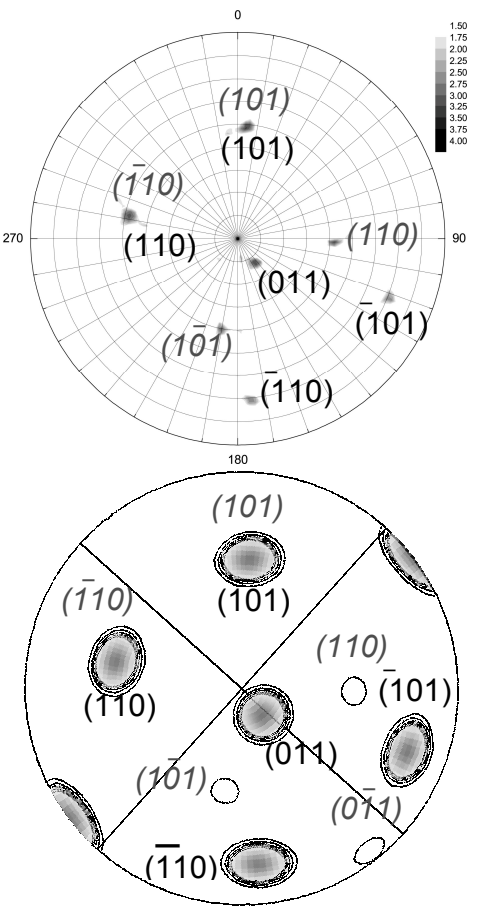

(111)fcc

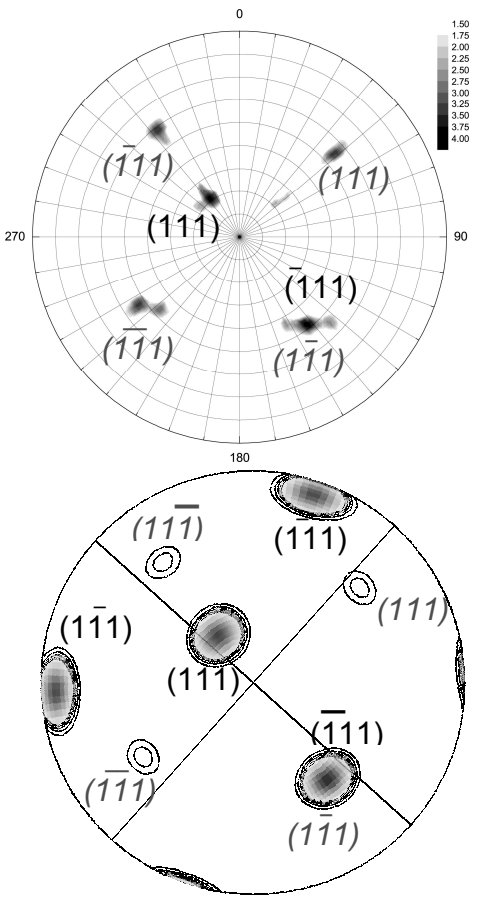

Fig. 2. XRD and EBSD pole figures (001), (101) and (111) as obtained for the austenite phase of the Bridgman crystal. The poles corresponding to the minor orientation are labelled in italics.

Although both phases in the Bridgman crystal have almost single-crystalline character, a second crystallographic orientation was found for austenite both in XRD and EBSD pole figures. The respective poles are labelled by italics indices in Figs. 2. The second crystallographic orientation could be described by the $\langle 001\rangle_{\text {fcc }}$ direction that was slightly inclined from the perpendicular direction to the sample surface. The sample surface perpendicular direction corresponds to the crystallographic direction $\langle 106\rangle$. Furthermore, a broadening and a "fine structure" of the poles in the XRD pole figures of the austenite was observed, which can be understood as a first indicator of local lattice rotations within austenite that is usually a consequence of the formation and organisation of microstructure defects. Such information could not obtained from the EBSD measurement, as the size of the poles in the pole figures calculated from the EBSD measurements is arbitrary. 


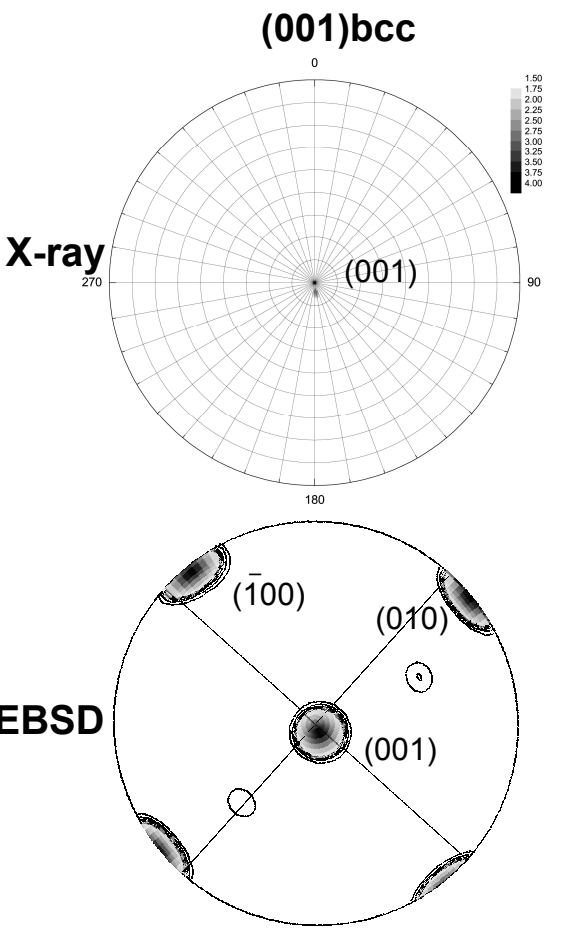

(101)bcc

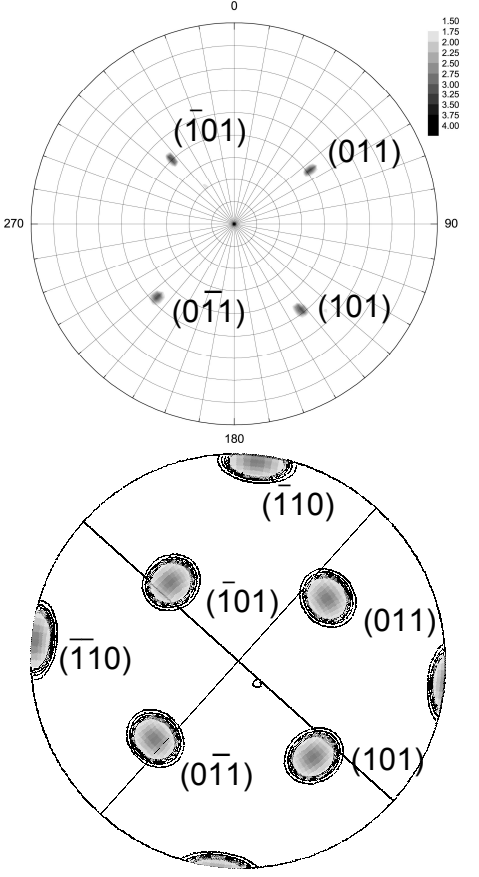

(111)bcc

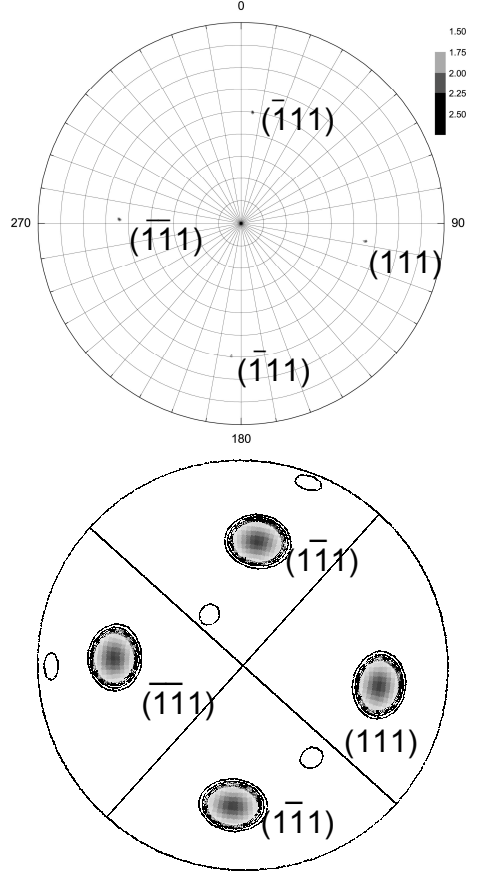

Fig. 3. XRD and EBSD pole figures (001), (101) and (111) as obtained for the ferrite from the Bridgman crystal.

The higher density of microstructure defects in austenite in comparison with ferrite was confirmed by XRD experiments that were carried out as $\Omega$ and $2 \theta / \Omega$ scans (Fig. 4 ). The $\Omega$ scans are performed at different inclinations of the sample from the symmetrical position and at a fixed detector position. The width of the $\Omega$ scans reveals the misorientation of individual grains. During $2 \theta / \Omega$ scans, the detector position is changed, but the direction of the diffraction vector with respect to the sample surface is constant. The width of this scan contains information on the distribution of the interplanar spacings. It can be inferred from the broad and partially split maxima in the $\Omega$ scans of austenite (Fig. 4) that the mean misorientations between individual crystallites exceed $2^{\circ}$.
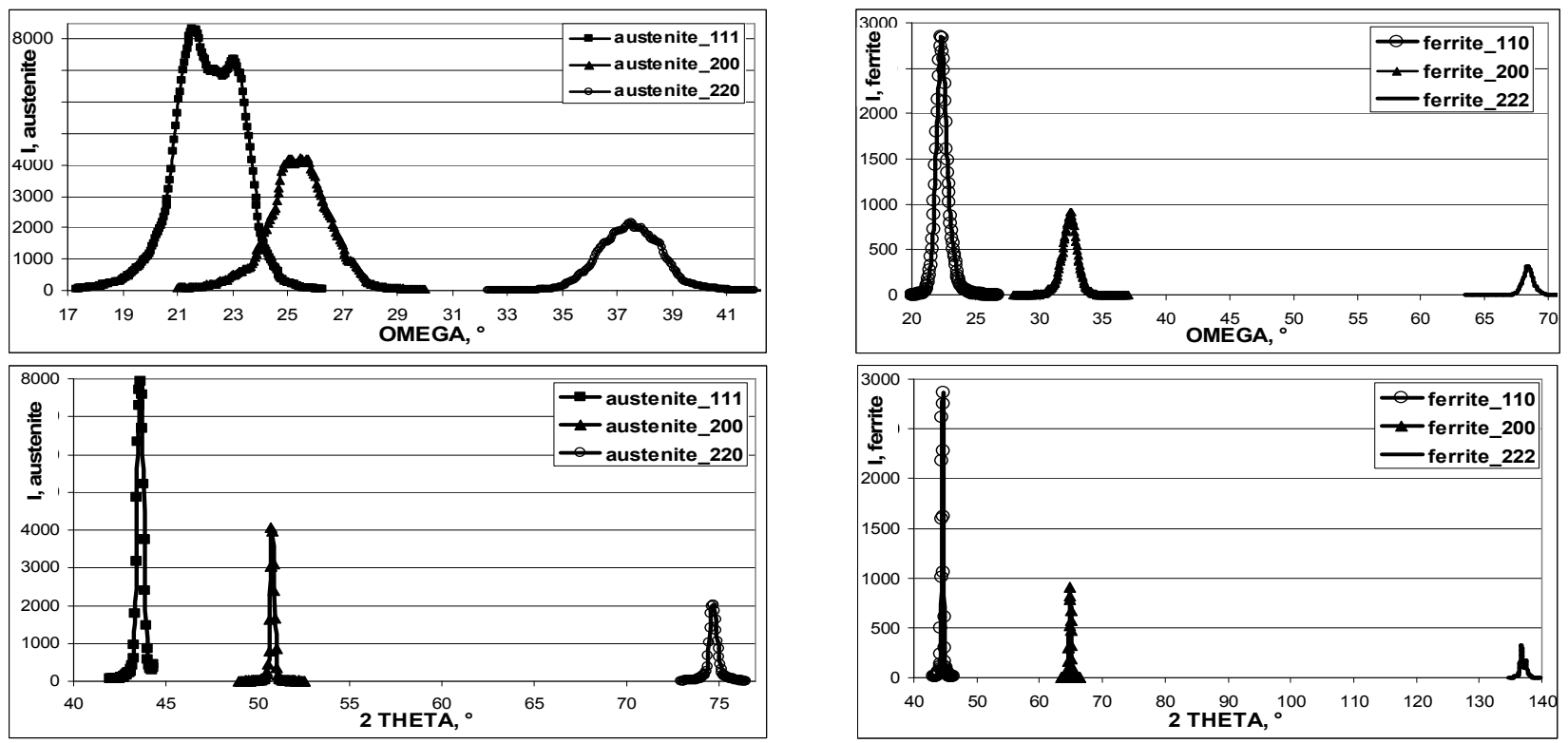

Fig. 4. Results of the $\Omega$ (upper row) and $2 \theta / \Omega$ scans (bottom) performed on different reflections of austenite (left) and ferrite (right). For an easier demonstration of the measured data, the $\Omega$ scans were shifted that the centres of gravity are located at the half of the diffraction angle $(2 \theta)$.

The $\Omega$ scans of ferrite are much narrower, indicating a smaller density of the microstructure defects, responsible for local lattice rotations. Austenite has also a higher density of microstructure 
defects compared to ferrite, which cause inhomogeneous variations of the interplanar spacing as it can be seen on the different broadening of XRD lines measured in the $2 \theta / \Omega$ mode. Reciprocal space mapping (RSM) performed on the diffraction line $200_{\mathrm{fcc}}$ (Fig. 5) revealed that a local lattice rotation in austenite has a stronger effect than the change of interplanar spacings, as the width of the intensity maximum is much larger in the $\mathrm{q}_{\mathrm{x}}$ direction than in the $\mathrm{q}_{\mathrm{z}}$ direction.

To analyse the microstructure defects in austenite that are responsible for the phenomena observed by XRD, particularly the large broadening of the intensity maxima in the $\Omega$ and $\mathrm{q}_{\mathrm{x}}$ directions (see Figs. 4 and 5), the phase boundary between ferrite and austenite was investigated using (HR)TEM (Fig. 6). A TEM image of the phase boundary is shown in Fig. 6a. The right grain (austenite) has a higher density of micro-twins than the left grain (ferrite), most likely due to lower stacking fault energy and a higher number of active slip systems in austenite. The dark fringes in austenite are due to long range distortion fields arising at interfaces between austenite and ferrite.

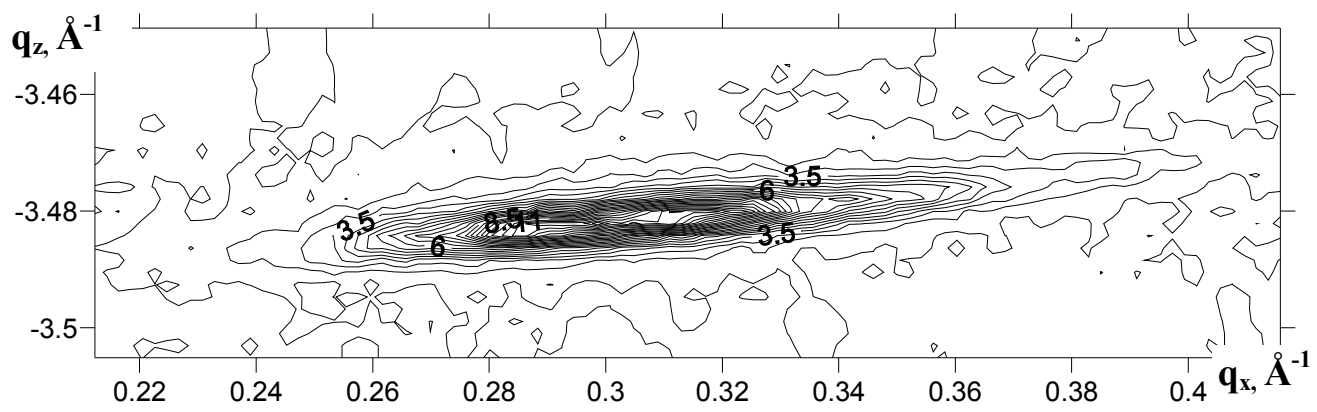

Fig. 5. Result of the reciprocal space mapping plotted in the $q_{x}-q_{z}$ coordinates of the one of the reciprocal space spots from the $\{001\}$ pole figures of austenite.
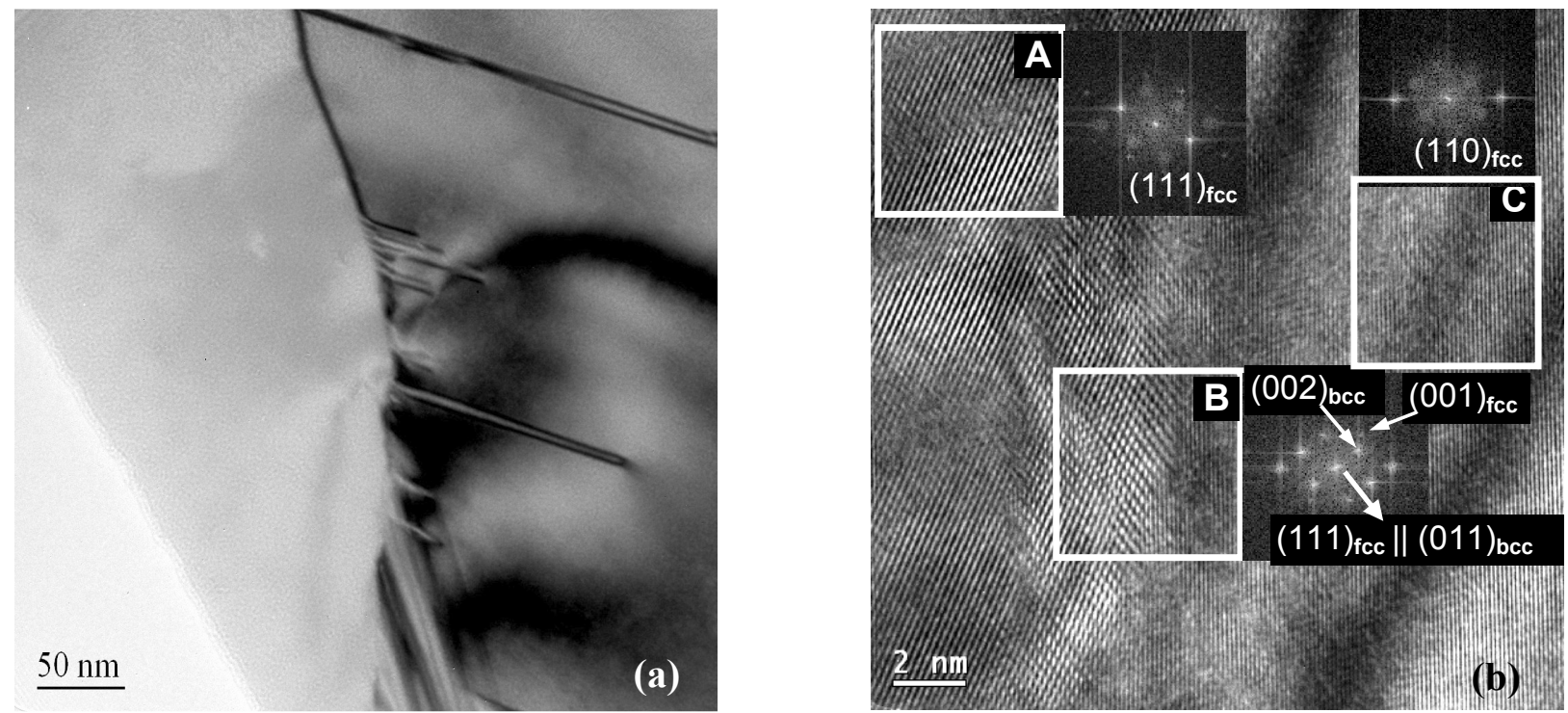

Fig. 6. (a) Transmission electron micrograph of a phase boundary between ferrite (left-hand side of the micrograph) and austenite (right-hand side of the micrograph) taken in diffraction contrast. Multiple twinning areas are apparent in the austenite; the micro-twins start at the phase boundary. (b) HRTEM image of two differently oriented austenite regions (A and $\mathrm{C}$ ) separated from each other by a narrow ribbon of ferrite (a part of the region B). The lattice planes (111) fcc and (011) $)_{\text {bcc }}$ are parallel to each other; the angle between the (002) planes of austenite and ferrite is approximately $10^{\circ}$.

HRTEM in conjunction with the Fast Fourier Transform of the HRTEM micrograph (Fig. $6 \mathrm{~b})$ confirmed the orientation relationship between ferrite and austenite obtained from the XRD and EBSD pole figures (Fig. 2), i.e., $(111)_{\mathrm{fcc}} \|(011)_{\mathrm{bcc}}$, and which is described by the NishiyamaWassermann model $[8,9]$. Further, HRTEM revealed the origin of the large local lattice rotations observed in austenite via XRD experiments (see i.e. Fig. 2, breadth of $\Omega$ scans in Fig. 4, broadening of diffraction maxima in the RSM along $\mathrm{q}_{\mathrm{x}}$ in Fig. 5 and the related discussions). HRTEM from Fig. 
$6 \mathrm{~b}$ shows two austenite regions with slightly different crystallographic orientations, separated from each other by a narrow, approximately $2 \mathrm{~nm}$ wide ribbon of ferrite. Thus, it can be surmised that such remnants of ferrite in austenite are responsible for the local lattice rotations in austenite. The FFT of HRTEM in Fig. 6b show that the lattice planes (002) of ferrite are tilted approximately $10^{\circ}$ to the planes (002) of austenite. More precisely, the angle between (002) bcc and (002) fcc planes was calculated using transformation matrices [12] to be equal to $9.7^{\circ}$. It might be possible that the tilt of the lattice planes (002) of ferrite against the lattice planes (002) of austenite assists in the formation of two austenite orientations, which were already observed in the XRD and EBSD pole figures (see Fig. 2). Furthermore, the thin slabs of ferrite separating two austenitic regions from each other seem to serve as a transition zone, promoting the development of such microstructure defects in austenite.

\section{Conclusions}

The Bridgman crystal of the TRIP steel under study consisted of austenite and ferrite. These two phases were interrelated by a Nishiyama-Wassermann orientation relationship, as obtained from the EBSD and XRD pole figures and confirmed by the HRTEM analysis. Whereas the ferrite was almost free of microstructure defects, a relatively high density of microstructure defects was found in austenite. The defects caused a mutual misorientation of crystallites in austenite as seen in several complementary XRD measurements. XRD pole figures reveal a second orientation of the austenite crystallites. The $\Omega$ scans and the reciprocal space maps showed a large broadening of diffraction maxima (the reciprocal space maps were broadened in the $\mathrm{q}_{\mathrm{x}}$ direction). TEM and HRTEM helped to elucidate the origin of the local lattice rotations in austenite. TEM revealed formation of complex micro-twin structures at the boundaries of neighbouring phases. HRTEM discovered tiny strips of ferrite between the austenitic regions with different crystallographic orientations. These remnants of ferrite were discussed to be related to the lattice rotation in austenite.

\section{Acknowledgements}

This work was supported by the German Research Foundation (DFG) in frame of the Collaborative Research Centre (SFB) 799. The authors thank to the Stahlzentrum Freiberg e.V. and Doncasters Precision Casting GmbH (Bochum) for providing us with the samples of the Bridgman crystals of TRIP steels. The HRTEM JEM 2010 FEF was purchased from the funds of the DFG priority programme SPP 1062.

\section{References}

[1] V. Uthaisangsuk, U. Prahl and W. Bleck: Computational Materals Science Vol. 45 (2009), p. 617-623

[2] U. Prahl, S. Papaefthymiou, V. Uthaisangsuk, W. Bleck, J. Sietsma and, S. van der Zwaag: Computational Materials Science Vol. 39 (2007), p. 17-22

[3] V. Uthaisangsuk, U. Prahl and W. Bleck: Computational Materials Science Vol. 43 (2008), p. 27-35

[4] S. Zaefferer, J. Ohlert and W. Bleck: Acta Materialia Vol. 52 (2004), p. 2765-2778

[5] H. Sato and S. Zaefferer: Acta Materialia Vol. 57 (2009), p. 1931-1937

[6] E.C. Bain: Trans. AIME Vol. 70 (1924), p. 25

[7] G. Kurdjumov and G. Sachs: Z. Physik Vol. 64 (1930), p. 325

[8] Z. Nishiyama: Sci. Rep. Tohoku Univ. Tokyo Vol. 23 (1934), p. 637

[9] G. Wassermann: Mitt. Kaiser-Wilhelm-Inst. Eisenforsch. Vol. 17 (1935), p. 149

[10] P. Haasen: Physical Metallurgy, $3^{\text {rd }}$ enlarged and revised edition, (Cambridge University Press 1996)

[11] P.W. Bridgman: U.S. patent office, 1,793,672. (1931)

[12] H. Schumann: Kristallgeometrie (VEB Deutscher Verlag für Grundstoffindustrie, Leipzig 1979). 